\title{
Analyse de miR-l et de sa cible potentielle Multiplexin dérégulés dans la dystrophie myotonique type 1
}

\author{
Anissa Souidi, Monika Zmojdzian, Émilie Plantié, Yoan Renaud, Coralie Gimonnet, \\ Krzysztof Jagla
}

La dystrophie myotonique de type 1 (DM1), une des maladies neuromusculaires les plus fréquentes chez l'adulte, à transmission autosomique dominante, est une maladie multisystémique caractérisée par une myotonie, une cataracte, une atrophie testiculaire et des troubles cardiaques tels que les complications de conduction, les arythmies et la cardiomyopathie dilatée. La DM1 est causée par une expansion de répétitions CTG dans la région 3'UTR du gène Dmpk (dystrophia myotonica protein kinase). Le nombre de ces répétitions varie entre 5 et 37 chez les personnes saines, et entre 50 et 4000 chez les patients DM1 [1]. Les transcrits mutés Dmpk forment des foci nucléaires qui séquestrent MBNL1 (Muscleblind-like 1) [2] et stabilisent CELF1 (ElavLike Factor 1) [3], deux facteurs d'épissage alternatif qui se fixent sur les régions 3'UTR de leurs gènes cibles. MBNL1 régule la maturation de miR-1, un microARN conservé, important pour la cardiogenèse et connu pour réguler des gènes codant des canaux potassiques et calciques dans le cour [4]. Il est fortement diminué dans les modèles DM1 de drosophile et aussi dans les muscles squelettique et cardiaque des patients DM1 [5]. L'objectif de notre étude est d'appliquer le modèle drosophilien pour effectuer une analyse fonctionnelle de miR-1 et de sa cible potentielle Multiplexin et de leurs implications dans les défauts cardiaques associés à la DM1. Mp, orthologue du collagène XV/XVIII chez les mammifères, est fortement dérégulée dans la DM1. Tout d'abord nous avons analysé l'effet de l'inhibition de miR-1 au niveau du coeur d'une part sur la structure et la physiologie cardiaque par l'approche SOHA (Semi-intact Optical Heartbeat Analysis) et d'autre part sur l'expression de Mp par immunomarquage. Ensuite nous avons analysé la fonction de Mp dans la physiologie cardiaque en utilisant des lignées $M p$ RNAi. Les résultats montrent une augmentation de l'expression de Mp dans le contexte DM1 et une diminution de la taille du coeur et des arythmies cardiaques dans le contexte perte de fonction $\mathrm{Mp}$, suggérant l'importance de la régulation de Mp dans le maintien de la structure et de la physiologie cardiaque et son implication probable dans les complications cardiaques associées à la DM1. La perte de fonction miR-1 au niveau cardiaque cause une cardiomyopathie dilatée accompagnée d'une augmentation de l'expression de $\mathrm{Mp}$, similaire aux symptômes DM1. L'ensemble des résultats suggèrent que la dérégulation de $\mathrm{Mp}$, dépendante de miR-1, contribue aux défauts cardiaques observés dans la DM1.

Analysis of miR-1 and its potential target Multiplexin deregulated in myotonic dystrophy type 1

\section{LIENS D'INTÉRÊT}

Les auteurs déclarent n'avoir aucun lien d'intérêt concernant les données publiées dans cet article.

\section{RÉFÉRENCES}

1. Meola G, Cardani R. Myotonic dystrophies: an update on clinical aspects, genetic, pathology, and molecular pathomechanisms. Biochim Biophys Acta 2015 ; 1852 : 594-606.

2. Mankodi A, Urbinati CR, Yuan QP, et al. Muscleblind localizes to nuclear foci of aberrant RNA in myotonic dystrophy types 1 and 2. Hum Mol Genet 2001; $10: 2165-70$.

3. Kim YK, Mandal M, Yadava RS, et al. Evaluating the effects of CELF1 deficiency in a mouse model of RNA toxicity. Hum $\mathrm{Mol}$ Genet $2014 ; 23: 293-302$.

4. Rau F, Freyermuth F, Fugier C, et al. Misregulation of miR-1 processing is associated with heart defects in myotonic dystrophy. Nat Struct Mol Biol 2011; $18: 840-5$.

5. Fernandez-Costa M, Garcia-Lopez A, Zuñiga S, et al. Expanded CTG repeats trigger miRNA alterations in Drosophila that are conserved in myotonic dystrophy type 1 patients. Hum Mol Genet 2013; 22 : 704-16. 


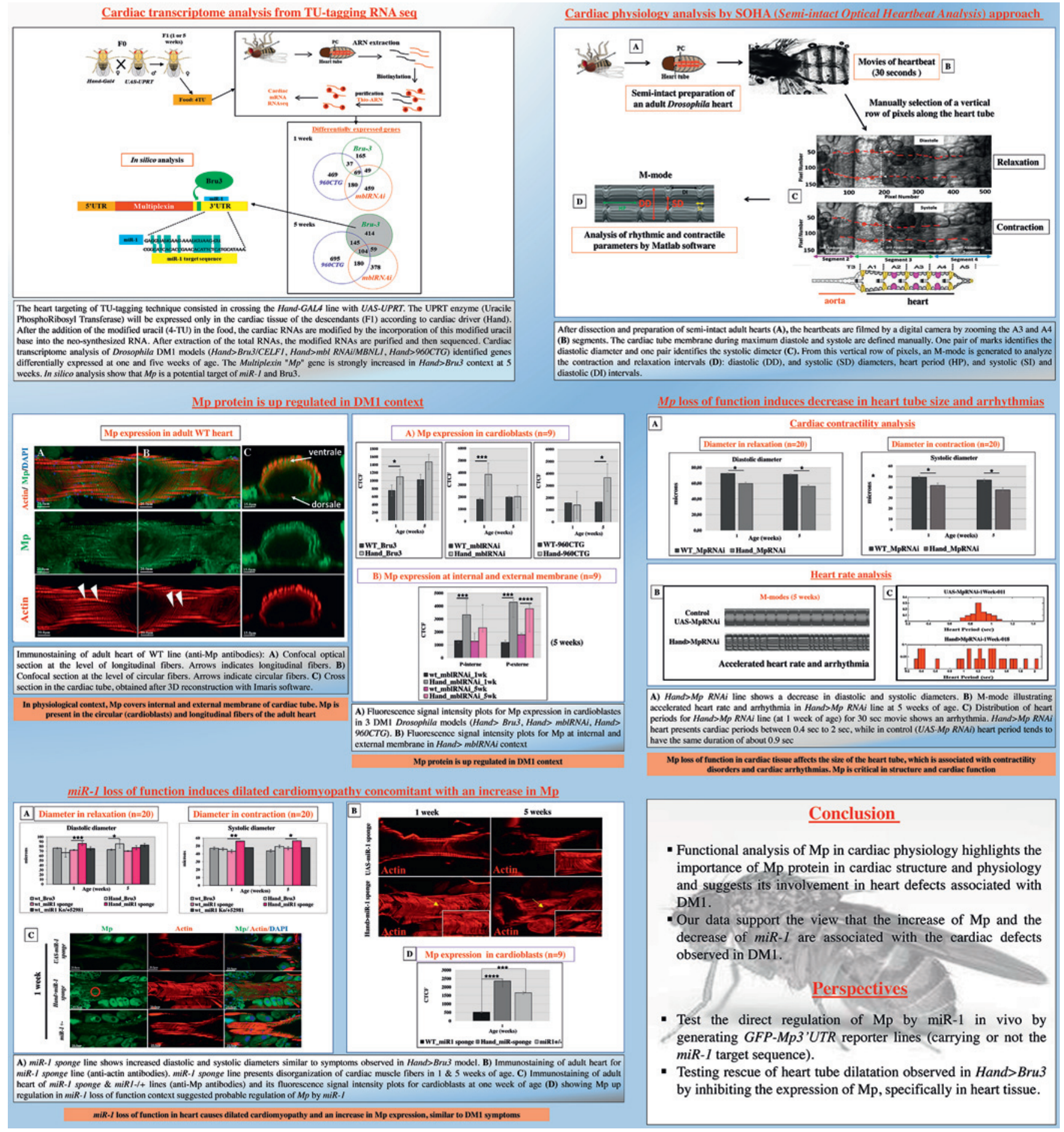

